\title{
Correlation of autologous skeletal myoblast survival with changes in left ventricular remodeling in dilated ischemic heart failure
}

Patrick I. McConnell, MD, ${ }^{\text {a }}$ Carlos L. del Rio, BS, ${ }^{\text {b }}$ Douglas B. Jacoby, PhD, ${ }^{d}$ Martina Pavlicova, PhD, ${ }^{c}$

Pawel Kwiatkowski, MD, ${ }^{a}$ Agatha Zawadzka, MS, ${ }^{d}$ Jonathan H. Dinsmore, PhD, ${ }^{d}$ Louis Astra, MD, ${ }^{a}$ Sheik Wisel, PhD,

and Robert E. Michler, MD ${ }^{\mathrm{a}}$

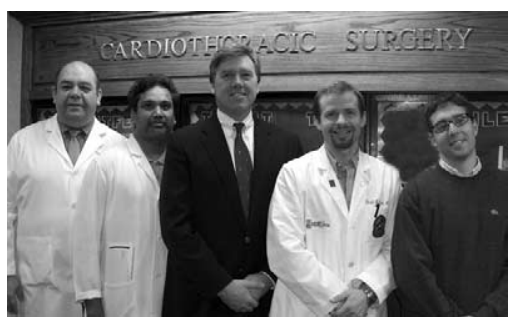

Kwiatkowski, Wisel, Michler, McConnell, del Rio

See related editorial on page 966 .

\section{Additional material is available online.}

From the Departments of Surgery, ${ }^{a}$ Anesthesiology, ${ }^{\mathrm{b}}$ and Statistics, ${ }^{\mathrm{c}}$ The Ohio State University, Columbus, Ohio, and GenVec Inc, ${ }^{\mathrm{d}}$ Charlestown, Mass.

D.B.J., J.H.D., and A.Z. are employees of GenVec, Inc. P.I.M. was supported in part by a Post Doctoral Fellowship from the Ohio Valley Affiliate, American Heart Association.

Read at the Thirtieth Annual Meeting of The Western Thoracic Surgical Association, Maui, Hawaii, June 23-26, 2004.

Received for publication Aug 10, 2004; revisions received Jan 25, 2005; accepted for publication Feb 10, 2005.

Address for reprints: Robert E. Michler, MD, John and Jean McCoy Professor of Surgery, The Ohio State University Medical Center, Division of Cardiothoracic Surgery, 400 West 10th Ave, N847 Doan Hall, Columbus, OH 43210 (E-mail: michler.1@ osu.edu).

J Thorac Cardiovasc Surg 2005;130:1001-9

$0022-5223 / \$ 30.00$

Copyright (C) 2005 by The American Association for Thoracic Surgery

doi:10.1016/j.jtcvs.2005.02.030
Objectives: The effect of autologous skeletal myoblast transplantation has not been rigorously studied in the setting of end-stage ischemic heart failure free of concomitant coronary revascularization. The aims of the present study were to determine autologous skeletal myoblast survival and its effects on left ventricular function and remodeling in sheep with dilated ischemic heart failure.

Methods: Ischemic heart failure (left ventricular ejection fraction, $30 \% \pm 2 \%$; left ventricular end-systolic volume index, $\left.82 \pm 9 \mathrm{~mL} / \mathrm{m}^{2}\right)$ was created in sheep $(\mathrm{n}=$ 11) with serial left circumflex coronary artery microembolizations. Instruments were inserted for the long-term determination of left ventricular global and regional dimensions, hemodynamics, and pressure-volume analysis after autologous skeletal myoblast transplantation (approximately $3.0 \times 10^{8}$ myoblasts; heart failure plus autologous skeletal myoblast group, $\mathrm{n}=5$ ) or without (heart failure-control group, $\mathrm{n}=6$ ). Measurements were performed in conscious animals.

Results: Autologous skeletal myoblast-derived skeletal muscle was found in all injected animals at 6 weeks. In ischemic heart failure, autologous skeletal myoblast cardiomyoplasty failed to improve systolic (left ventricular ejection fraction, 29\% \pm $4 \%$; $\mathrm{dP} / \mathrm{dT}_{\max }, 2863 \pm 152 \mathrm{~mm} \mathrm{Hg} / \mathrm{s}$; end-systolic elastance, $1.6 \pm 0.22$ ) or diastolic (left ventricular end-diastolic pressure, $21 \pm 2 \mathrm{~mm} \mathrm{Hg}$; time constant of relaxation (Tau), $34 \pm 4 \mathrm{~ms}$; $\mathrm{dP} / \mathrm{dT}_{\min },-1880 \pm 68 \mathrm{~mm} \mathrm{Hg} / \mathrm{s}$ ) function. There was, however, attenuation in the left ventricular dilatation after autologous skeletal myoblast transplantation (change in end-systolic volume index, $14 \% \pm 4 \%$ vs $32 \%$ $\pm 6 \% ; P<.05)$. The effects of autologous skeletal myoblast-derived skeletal muscle were exclusive to the left ventricular short-axis dimension and dependent on autologous skeletal myoblast survival $\left(R^{2}=0.59, P=.006, \mathrm{n}=11\right)$.

Conclusions: Autologous skeletal cardiomyoplasty was able to attenuate left ventricular remodeling in sheep with end-stage ischemic heart failure.

$\Delta$ utologous skeletal myoblast (ASM) transplantation, or cardiomyoplasty, has been shown in multiple experimental studies to improve cardiac function after myocardial infarction. ${ }^{1-7}$ Although the majority of studies have been performed in small-animal models of myocardial infarction, there is evidence of similar improvement in models involving larger animals ${ }^{8}$ and in the first patient trials. ${ }^{9-12}$ The mechanism behind such positive functional changes remains poorly understood given that developing and engrafted skeletal myoblasts are electromechanically isolated from their host myocardium (as evidenced by the lack of connexin-43, gap junctions, or both). ${ }^{4,8-10,12}$ Furthermore, clinical ASM cardiomyoplasty has been applied exclusively to patients with severe ischemic cardiomyopathy, and more importantly, it has always been performed as an adjunct to coronary 
revascularization, placement of a left ventricular (LV) assist device, or both. ${ }^{11}$ Because of these concomitant therapies, the improvements in indices of myocardial perfusion, viability, and function might be difficult to attribute to ASM injection alone.

Additionally, growing experimental evidence suggests that the number of ASM cells transplanted and the functional-geometric effects are directly related. ${ }^{5,12}$ For example, Tambara and associates, ${ }^{5}$ using fetal-derived ASM in rats, demonstrated that both cardiac function and remodeling were affected in a dose-dependent fashion. However, these benefits have not been demonstrated in ischemic dilated heart failure (HF), where increased wall stresses and altered myocardial mechanoenergetics could compromise ASM survival, differentiation, and ultimately functional efficacy. Thus, the aims of the present study were to evaluate LV remodeling and function after ASM transplantation in an animal model of end-stage ischemic HF (LV ejection fraction $[\mathrm{LVEF}]$ of $<35 \%$ and $\mathrm{LV}$ end-systolic volume of $>80 \mathrm{~mL} / \mathrm{m}^{2}$ ). Furthermore the study also sought to evaluate the survival, differentiation, and alignment of ASMs injected in those same animals.

\section{Materials and Methods}

All experiments were approved by The Ohio State University Institutional Laboratory Animal Care and Use Committee and comply with published federal guidelines.

\section{Ischemic HF Model}

Experimental ischemic HF was created in sheep in the same way as Sabbah and colleagues ${ }^{13}$ described in dogs, with minor modifications. In brief, serial and selective left circumflex coronary artery (LCxA) microembolizations $(2.9 \pm 0.4$ injections per animal) were performed by injecting polystyrene beads $(70-110 \mu \mathrm{m})$ weekly until the LVEF was maintained at or less than $35 \%$ for 2 consecutive weeks.

\section{Experimental Groups}

The HF control group of sheep (baseline) was instrumented 2 weeks before LCxA microembolization and HF induction (HF control, $n=6$ ). The transplanted group of sheep had LCxA microembolization and $\mathrm{HF}$ induction before instrumentation and injection with ASMs (HF plus ASM, $n=5$ ). Studies were performed weekly for 6 weeks in awake and unsedated animals.

\section{Instrumentation for Long-Term Monitoring}

All sheep were instrumented through a left thoracotomy; an LV solid-state electronic pressure transducer $(4.0$ or $4.5 \mathrm{~mm}$, Konigsberg Instruments, Pasadena, Calif) was placed into the left ventricle at its apex, and permanent, heparinized $(1000 \mathrm{U} / \mathrm{mL})$, fluidfilled catheters (Tygon; Norton Performance Plastics, Akron, Ohio) were inserted for monitoring of aortic, LV, and right ventricular pressures. Six piezoelectric crystals (Sonometrics Inc, New London, Ontario, Canada) were surgically placed in the LV endocardium at the midpapillary level (short axis [SA]), at the LV base and apex (long axis [LA]), and in the midmyocardium of the posterolateral left ventricle (segment length $\left[\mathrm{SL}_{\text {post }}\right]$ ). A $16-\mathrm{mm}$ occluder (In Vivo Metrics, Healdsburg, Calif) was positioned around the inferior vena cava. All catheters and cables were tunneled to positions between the animals' scapulas.

\section{Hemodynamic Measurements and Pressure-Volume Analysis}

Aortic, right ventricular, and LV fluid-filled catheters were attached to calibrated Statham pressure transducers (Model P23XL; Biggo-Spectramed, Oxnard, Calif) and amplified (Gould, Valley, Ohio). The electronic LV pressure gauge was calibrated by using the LV fluid-filled catheter. Pressures waveforms were collected (at $1 \mathrm{kHz}$ ) and analyzed by using a 16-channel data acquisition and software system (IOX; EMKA Technologies, Falls Church, Va).

Sonometric signals were analyzed for waveform cardiac-cycle dependent (end-diastolic and end-systolic) and independent (eg, minimum, maximum, and mean) parameters. LV volume (in milliliters) was calculated in real time by using SA and LA dimensions and the following equation: $\left(S A^{2} * L A * \pi / 6\right) / 1000 .{ }^{14} \mathrm{LV}$ volume indices were calculated as follows: $L V$ volume*Body surface area $\left(\mathrm{mL} / \mathrm{m}^{2}\right)$. Inferior vena caval occlusions were performed for the generation of pressure-volume (PV) relationships that were analyzed offline with analysis software (IOX, EMKA Technologies).

For information on skeletal muscle biopsy and ASM culture and histology, see Appendix E1.

\section{Statistical Analysis}

Data are presented as means \pm standard error of the mean. The differences between groups (treatments $=$ HF plus ASM and HF-control) over time for LV hemodynamic, geometric, and functional data were studied with multifactorial (2-way) analysis of variance with repeated measurements (factors $=$ group and time). If the $\mathrm{F}$ ratio exceeded a critical value $(\alpha<.05)$, the post-hoc Student-Newman-Keuls method was used to perform pairwise comparisons (SigmaStat; Systat Software, Inc, Point Richmond, Calif).

Individual PV relationships were computed by means of regression analysis (IOX, EMKA Technologies). Additionally, the equality of the PV relationships between the HF plus ASM and HF-control groups was studied with multiple linear regression considering both qualitative (group) and interaction terms (ie, simultaneously testing the differences in slope and intersect of the regression functions; Minitab R14; Minitab, Inc, State College, $\mathrm{Pa}$ ). Linear regression analyses were also performed to study the relationship-interaction between indices of $\mathrm{LV}$ remodeling or function and the estimated number of surviving ASM-derived myocytes, including HF-controls as zero survival (Minitab R14, Minitab, Inc).

The differences in the same control animals before HF (baseline) and after HF (week 1) were studied by a paired Student $t$ test (SigmaStat, Systat Software, Inc) to validate the impaired physiology of HF present in this model. However, because HF was defined as both increased end-systolic volume index (ESVI) and decreased LVEF (a null hypothesis consisting of 2 variables), the Bonferroni method for multiple comparisons was used to correct the level of confidence $(\alpha<.025)$. 

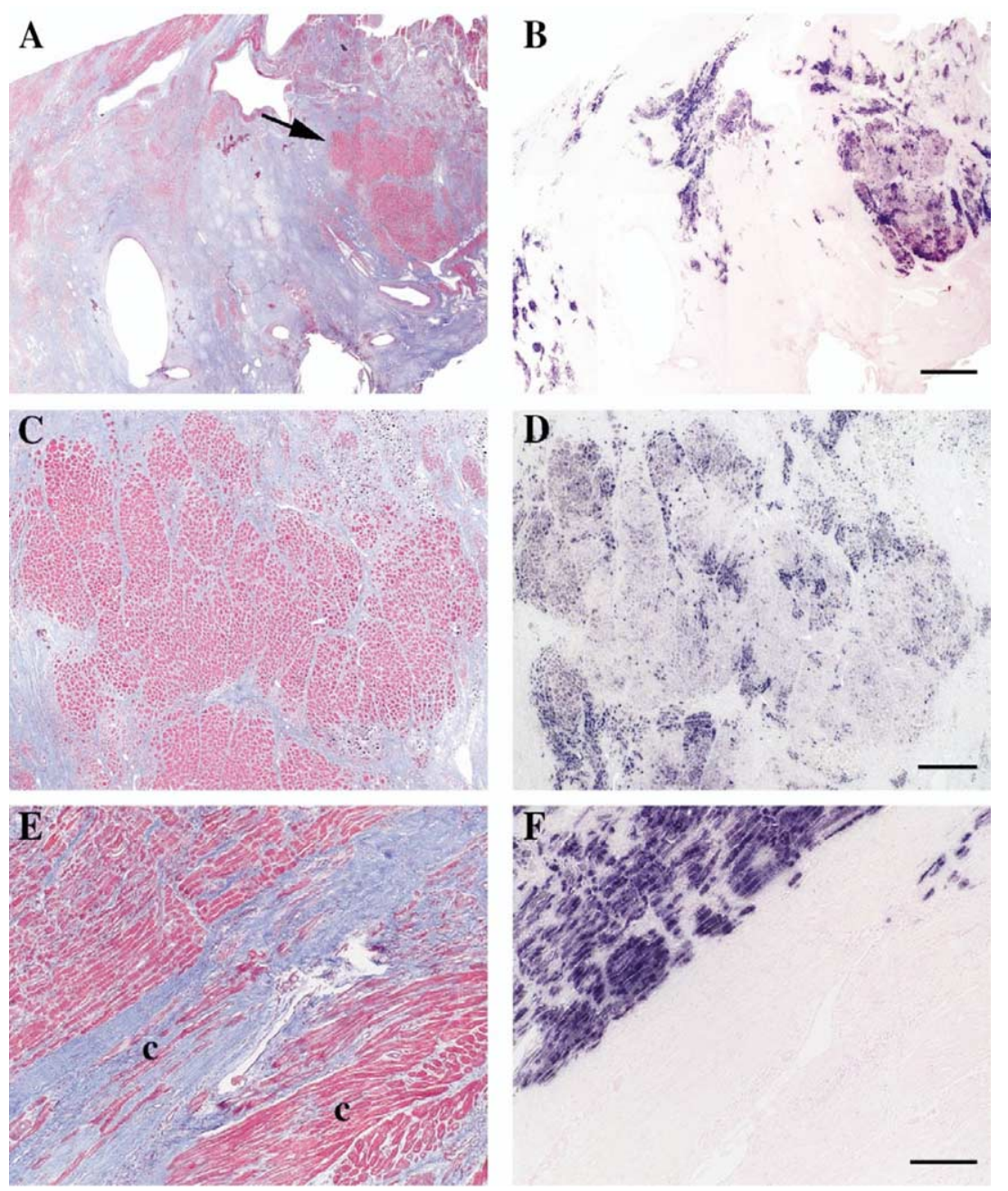

Figure 1. Six weeks after ASM injection in sheep with ischemic HF, composite Trichrome (A) and skeletal muscle-specific myosin heavy chain (B; MY-32, purple staining)-stained sections demonstrate extensive patches of ASM-derived skeletal muscle fibers engrafted in areas of myocardial scar. In $C$ and $D$, at higher magnification from panel $A$ (arrow), skeletal fibers were seen aligned with each other and further organized into myofibril bundles. ASM-derived skeletal muscle aligned with remaining cardiac myocytes $(E, c)$ and with neighboring skeletal myofibers, which was confirmed with staining for MY-32 (F). Scale bars in panels B, D, and F are $2 \mathrm{~mm}, 0.5 \mathrm{~mm}$, and $0.2 \mathrm{~mm}$, respectively.

\section{Results}

Eleven sheep were studied for 6 weeks after establishment of HF with (HF plus ASM, $\mathrm{n}=5$ ) or without (HF-control, $\mathrm{n}=6)$ ASM injection. Three of 8 sheep intended for the HF plus ASM group died during the instrumentation procedure, either before ASM injection $(n=2)$ or within 72 hours after injection, and were not included in the study. No sheep in the HF-control group died early. Sheep were less active after HF induction, but no differences in daily observations were appreciated between groups.

\section{Histology}

The average number of injected myoblasts was $3.44 \pm 0.49$ $\times 10^{8}$ cells, ranging from 1.53 to $4.3 \times 10^{8}$ cells. Myoblast purity $(92 \% \pm 1.4 \%)$ and cell viability $(93 \% \pm 1.2 \%)$ were assessed at the time of transport, and myoblast viability was confirmed to be greater than $90 \%$ (by using trypan blue exclusion) after shipment $\left(4^{\circ} \mathrm{C}\right)$. ASM-derived skeletal myofibers were found in all injected hearts, but the estimated survival (see discussion) of injected myoblasts sur- viving at week 6 ranged from 158,000 cells $(0.05 \%$ survival) to 36.4 million cells (10.7\% survival).

Representative histologic sections with detailed descriptions are found in Figure 1 and Figure E1. In general, skeletal myocytes were seen aligned with other skeletal muscle fibers, as well as aligned with remaining cardiac myocytes (Figure 1, $C-F$; Figure E1, $A$ and $B$ ). Engrafted skeletal muscle fibers were characterized by staining to the myosin heavy chain fast-twitch isoform (purple staining in Figure $1, B, D$, and $F$, and Figure E1, $B$ ). However, in no section were ASM-derived myofibers seen stained for troponin I or connexin-43, despite close apposition to surviving cardiac myocytes (Figure E1, $C$ and $D$, respectively).

\section{Cardiac Hemodynamics}

Hemodynamic data are summarized in Table 1 . No animal had improvement in $\mathrm{dP} / \mathrm{dT}_{\max }$ or LVEF after ASM injection. No linear relationship was found between the esti- 
TABLE 1. Cardiac hemodynamics after autologous skeletal myoblast transplantation

\begin{tabular}{|c|c|c|c|c|c|}
\hline & $\begin{array}{l}\text { Baseline } \\
(n=6)\end{array}$ & $\begin{array}{c}\text { HF-control, } \\
\text { week } 1(n=6)\end{array}$ & $\begin{array}{c}\text { HF-control, } \\
\text { week } 6(n=6)\end{array}$ & $\begin{array}{c}\text { HF+ASM, } \\
\text { week } 1(n=5)\end{array}$ & $\begin{array}{c}\text { HF+ASM, } \\
\text { week } 6(n=5)\end{array}$ \\
\hline \multicolumn{6}{|l|}{ Heart failure } \\
\hline $\mathrm{ESVI}, \mathrm{mL} / \mathrm{m}^{2}$ & $39 \pm 4$ & $93 \pm 7^{*}$ & $124 \pm 15 \dagger \ddagger$ & $85 \pm 16$ & $98 \pm 18 \ddagger$ \\
\hline LVEF, \% & $48 \pm 2$ & $30 \pm 2^{*}$ & $28 \pm 2$ & $29 \pm 4$ & $27 \pm 4$ \\
\hline HR, beats/min & $109 \pm 4$ & $126 \pm 7^{*}$ & $125 \pm 9$ & $128 \pm 9$ & $110 \pm 10 \dagger$ \\
\hline LVSP, mm Hg & $106 \pm 6$ & $103 \pm 5$ & $102 \pm 7$ & $103 \pm 2$ & $106 \pm 4$ \\
\hline LVEDP, mm Hg & $11 \pm 1$ & $23 \pm 4^{*}$ & $26 \pm 3$ & $21 \pm 2$ & $22 \pm 3$ \\
\hline $\mathrm{EDVI}, \mathrm{mL} / \mathrm{m}^{2}$ & $75 \pm 7$ & $135 \pm 9^{*}$ & $170 \pm 15 \dagger \ddagger$ & $118 \pm 16$ & $131 \pm 21 \ddagger$ \\
\hline $\mathrm{dP} / \mathrm{dT}_{\text {max }}, \mathrm{mm} \mathrm{Hg} / \mathrm{s}$ & $3414 \pm 92$ & $2428 \pm 327^{*}$ & $1864 \pm 216 \dagger$ & $2863 \pm 152$ & $2166 \pm 174 \dagger$ \\
\hline $\mathrm{dP} / \mathrm{dT}_{\min }, \mathrm{mm} \mathrm{Hg} / \mathrm{s}$ & $-2124 \pm 108$ & $-1582 \pm 173^{*}$ & $-1423 \pm 147$ & $-1880 \pm 68$ & $-1713 \pm 84$ \\
\hline Tau, ms & $17 \pm 1$ & $38 \pm 8^{*}$ & $39 \pm 4$ & $34 \pm 4$ & $44 \pm 3 \dagger$ \\
\hline \multicolumn{6}{|c|}{$\begin{array}{l}\text { Pressure-volume analysis, } \\
\text { HF+ASM }(n=4), H F- \\
\text { control }(n=5)\end{array}$} \\
\hline $\mathrm{E}_{\mathrm{es}}$ & $3.7 \pm 0.5$ & $1.3 \pm 0.11^{*}$ & $0.9 \pm 0.13$ & $1.6 \pm 0.22$ & $1.6 \pm 0.28$ \\
\hline $\mathrm{V}_{0}$ & $18 \pm 3.9$ & $39 \pm 7.8^{*}$ & $59 \pm 8.7 \dagger \ddagger$ & $32 \pm 3.1$ & $25 \pm 3.4 \ddagger$ \\
\hline $\mathrm{M}_{\mathrm{w}}$ & $97 \pm 7.4$ & $66 \pm 10.1^{*}$ & $58 \pm 7.8 \dagger$ & $67 \pm 5.9$ & $57 \pm 8.0 \dagger$ \\
\hline$V_{w}$ & $50 \pm 9.5$ & $88 \pm 4.9^{*} \ddagger$ & $107 \pm 15 \dagger \ddagger$ & $75 \pm 3.3 \ddagger$ & $71 \pm 2.9 \ddagger$ \\
\hline
\end{tabular}

Mean \pm standard error of the mean. $H F$, Heart failure; $A S M$, autologous skeletal myoblast; $E S V I$, end-systolic volume index; $L V E F$, left ventricular ejection fraction; $H R$, heart rate; $L V S P$, left ventricular systolic pressure; $L V E D P$, left ventricular end-diastolic pressure; $E D V I$, end-diastolic volume index; $d P / d T$, derivative of pressure, $T a u$, time constant of relaxation (Weiss method); $E_{e s}$ end-systolic elastance; $V_{0,}$ x-intercept of end-systolic pressure volume; $M_{w}$, preload recruitable stroke work; $V_{w}$ x-intercept of preload recruitable stroke work. $* P<.05$ from baseline to HF-control (week 1 ). $\dagger P<.05$ from week 1 within groups (1-way analysis of variance). $\ddagger P<.05$ between groups at respective times (2-way analysis of variance).

mated number of surviving cells and LVEF $\left(R^{2}=0.00017\right.$, $P=.99)$ or $\mathrm{dP}_{\mathrm{dT}} \mathrm{dT}_{\max }\left(R^{2}=0.048, P=.543\right)$.

\section{PV Analysis}

Data for end-systolic pressure-volume relationship, preload recruitable stroke work (PRSW), and LV work (PVA) from HF-control and HF plus ASM sheep are summarized in Table 1 and exemplified in Figure E2. As expected and demonstrated by means of PV analysis, HF induction resulted in significant decreases in the slope of PRSW $\left(\mathrm{M}_{\mathrm{w}}\right)$ and in the load-independent contractility index $\left(\mathrm{E}_{\mathrm{es}}\right)$. No significant differences between treatment groups were observed at week 1 , with both presenting comparable degrees of dysfunction. Multiple linear regression analyses accounting for covariance between groups also demonstrated no significant differences in slope (week $1, P=.614$; week 6 , $P=.519$; power $=1$ ) or intercept (week $1, P=.945$; week $6, P=.928$; power $=1$ ) of the volume-adjusted $\mathrm{PV}$ relationships. No linear relationship was found between the estimated number of surviving cells and $\mathrm{E}_{\mathrm{es}}\left(R^{2}=0.088, P\right.$ $=.436)$ or $\mathrm{M}_{\mathrm{w}}\left(R^{2}=0.018, P=.731\right)$.

There was an increase (rightward shift, $P=.026$ ) in the $\mathrm{V}_{0}$ (x-intercept) of the $\mathrm{E}_{\mathrm{es}}$ for the HF-control group from week 1 to week 6 (Figure E2). The $\mathrm{V}_{0}$ tended $(P=.20)$ to decrease (leftward shift) over the 6 weeks in the HF plus ASM animals, and a difference was noted $(P=.014)$ between the HF-control and HF plus ASM animals at week 6 , supporting the hypothesis that ASM injection attenuated
LV remodeling. Likewise, the $\mathrm{x}$-intercept of the PRSW was increased from week 1 to week 6 in the HF-control group $(P$ $=.03)$ and remained different $(P=.009)$ compared with that seen in the HF plus ASM group at week 6 (Table 1 and Figure E2).

\section{Sonomicrometry and LV Segmental Function}

LV regional and segmental data are presented in Table E1. $\mathrm{HF}$ induction significantly $(P<.05)$ increased the segmental length in the infarct region $\left(\mathrm{SL}_{\text {post }}\right)$ of HF-control animals. Over the course of the study (week 1 to week 6), no significant differences were observed in $\mathrm{SL}_{\text {post }}$ for either group of animals. LV segmental dyskinesia was present after microembolization, and therefore both systolic bulging and postsystolic shortening were evident in both groups throughout the 6-week study.

\section{Sonomicrometry and LV Dimensions}

LVESVI and end-diastolic volume index were increased $(P$ $<.05$ ) from baseline in both groups at HF week 1; however, there was no difference between groups at week 1 (Table 1). In HF plus ASM animals LV dilatation was attenuated compared with that seen in HF-control animals $(P=.016)$ by week 3 (change in ESVI: $5.3 \% \pm 1.2 \%$ and $17.8 \% \pm$ $3.3 \%$, respectively), and this difference progressed $(P=$ .006 ) out to week 6 (Figure 2). The difference in LV volume resulted from a significant $(P=.005)$ attenuation in SA dilatation alone (Figure 2). No difference $(P>.05)$ was 

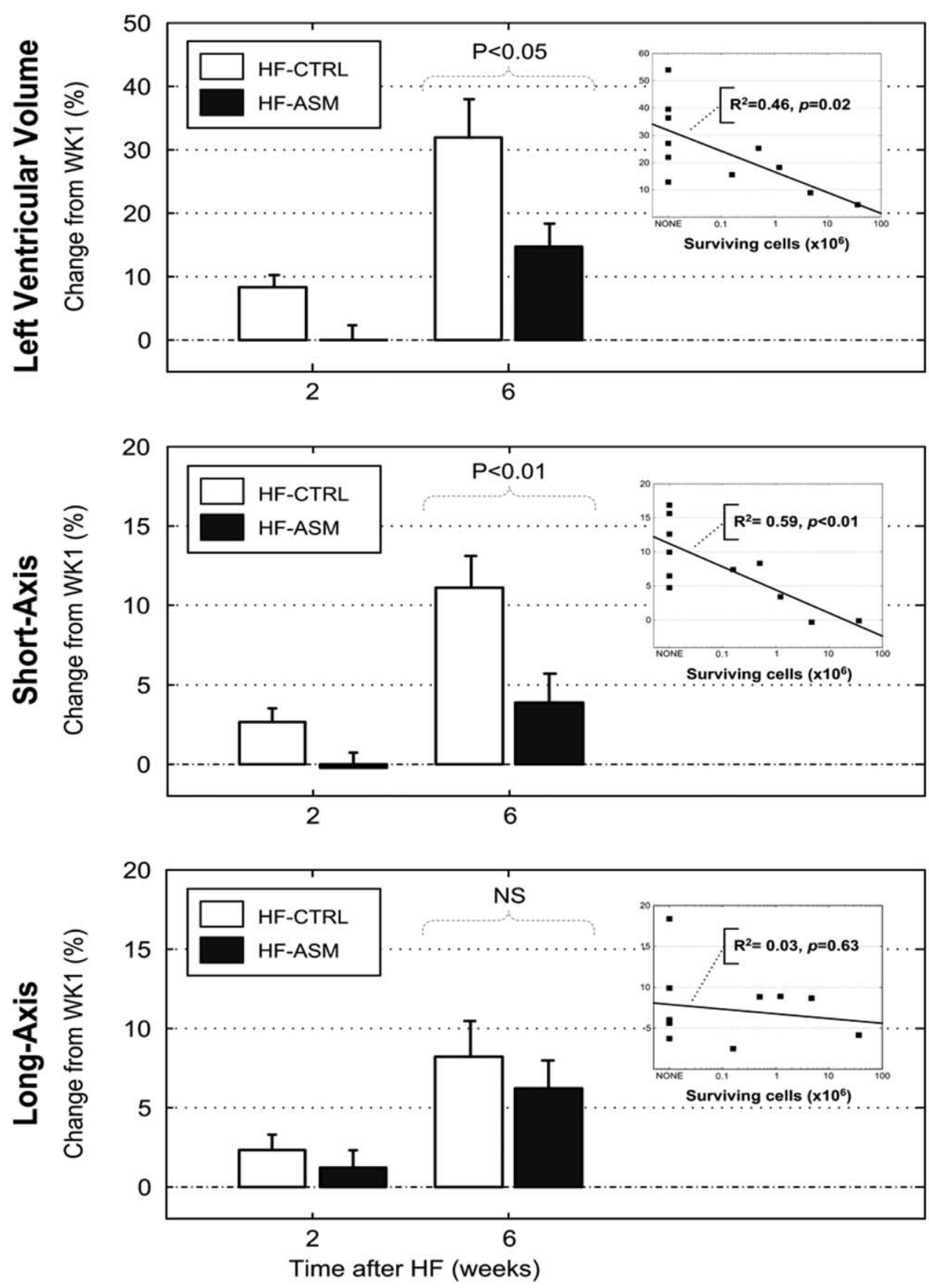

Figure 2. LV dilatation (ESVI, top pane/) and the increase in midpapillary short-axis length (SA, middle pane/) were attenuated after ASM injection ( $n=5$, open bars) compared with that seen in HF-control animals ( $n=6$, shaded bars). LV long-axis length (LA, bottom panel) was not different between groups. All animals, including HF-control animals, were used to evaluate the relationship of ASM-derived myocyte survival (log of surviving cells) compared with that of $L V$ remodeling (inset of each panel, $n=11$ ). Animals with the highest ASM-derived myocyte survival demonstrated the greatest attenuation, particularly in LV short-axis dilatation. Correlative statistics are presented for each relationship. 
found in LA dilatation between groups. Correlations of ESVI, SA, and LA to estimated ASM survival are presented in Figure 2.

\section{Discussion}

Few studies have examined the effect of ASM in hearts with a preexisting and clinically significant degree of ischemic dysfunction and remodeling (LVEF of $<35 \%$ with LVESVI of $>80 \mathrm{~mL} / \mathrm{m}^{2}$ ). The goal of the present study was to determine the therapeutic benefit of ASM cardiomyoplasty in a clinically applicable model of ischemic dilated HF free of the confounding factors associated with coronary revascularization or other supportive therapies.

ASM-derived skeletal muscle was found in all injected sheep at 6 weeks. As others have reported, ${ }^{4,7-12}$ no staining for connexin-43 was found in ASM-derived skeletal muscle. Transplanted ASM-derived skeletal myofibers aligned with each other and with the remaining cardiac myofibers in all sections (Figures 1 and E1). Such organized alignment of the ASM-derived fibers suggests that these fibers remained sensitive to stress-strain relationships found within the myocardium. ${ }^{15-17}$

A major limitation with cell therapy in general is the large percentage (up to 90\%) of cells that are lost shortly after injection. ${ }^{18,19}$ An explanation for this early loss might be by means of lymphatic drainage, venous drainage, or both, of the cells after direct intramyocardial injection. ${ }^{19}$ Other factors also likely contribute to the further loss of cells that are retained within the myocardium-scar. Recent investigations have shown that both the pretreatment ${ }^{20}$ and transfection $^{21}$ of ASM with vascular endothelial growth factor improved cardiac function, presumably by enhancing perfusion and nutrient delivery. Furthermore, strategies to limit inflammation, apoptosis, or both, have also proved beneficial to improving the efficacy after cellular cardiomyoplasty. ${ }^{22,23}$ However, we did not observe evidence for inflammation at graft sites 6 weeks after injection (Figures 1 and E1). Even with relatively low myoblast cell survival (Figure 1, animal with $1.1 \%$ cell survival), considerable areas of scarred myocardium can be filled with viable myofibers as a result of cell fusion and subsequent enlargement of myofibers (approximately a 10-fold increase in ASMderived myofiber cross-sectional area per nucleus vs myoblasts).

\section{Function}

Data evaluating cardiac performance after ASM injection in Tables 1 and E1 suggest no improvement in any hemodynamic parameter or index of cardiac contractility in sheep with end-stage, dilated ischemic HF. The lack of a demonstrable direct functional benefit observed in our study differs from reports in other animal models using a single ischemic insult (cryoinfarction, ${ }^{6}$ ligation, ${ }^{7}$ and coil embolization ${ }^{8}$ ) and might be related to the chronic nature and severity of LV dysfunction in our HF model (multiple microinfarctions over several weeks). Thus in the sheep HF model the insult might have more effectively exhausted remote myocardial compensatory mechanisms preventing contribution from the remote myocardium after ASM injection. This could explain that the discrepancy with results previously published in sheep (Ghostine and colleagues ${ }^{8}$ ) after coronary occlusion might be due to the worse LV structure and function in our sheep.

Other possible explanations for the lack of observed functional cardiac improvements include the extent of remodeling at the time of treatment, the methods used for cell preparation, and technical flaws. We agree with the interpretation offered by Jain and associates, ${ }^{7}$ in their ex vivo preparation in rats, that modest functional improvements observed after ASM injection were likely the result of benefits to nonfunctional properties of the left ventricle (ie, attenuated LV dilatation) rather than directly to LV contraction. In essence, less wall stress placed on remote cardiac myocytes as a result of ASM-derived skeletal muscle preventing further LV chamber dilatation would translate into better remote myocardial function. Perhaps the earlier the treatment, the sooner the benefits of ASM-derived skeletal muscle could be realized on LV remodeling and the greater the likelihood that the remote cardiomyocytes could adequately compensate and contribute to global LV function.

With respect to differences in the cell preparation in our study, the limited functional benefit of the ASM in our report could have resulted from the greater myoblast purity of our injectate, the method of cell expansion, cryopreservation, rewarming, and/or the transportation of the ASM. Unlike Pouzet and coworkers, ${ }^{12}$ who demonstrated in rats stratified for LV function (LVEF) a significant correlation with the number of cells injected to indices of LV function and that those most severely impaired received the greatest benefit, we were unable to demonstrate such a relationship compared with the number of surviving ASM-derived myocytes. Pouzet, ${ }^{12}$ Ghostine, ${ }^{8}$ and their colleagues present myoblast purity of less than $50 \%$ at the time of injection and showed improved systolic function, whereas we expanded a more pure population of myoblasts $\left(>90 \% \mathrm{CD}^{+} 6^{+}\right)$and found none. Fibroblasts, as the major contaminant in these cell preparations, on their own have not been reported to enhance systolic function, as has been reported for myoblasts. ${ }^{24}$ However, we cannot rule out synergistic effects between fibroblasts and myoblasts, which could account for improved contractility in preparations that are $50 \%$ versus $90 \%$ pure.

Other obvious differences in the expansion and storage of cells in our study include the cryopreservation and subsequent thaw of cells before implantation, as well as shipment at $4^{\circ} \mathrm{C}$. Histologically, we could not document any 
obvious differences in the contractile protein staining (MY32), inflammation, ASM coalignment, and alignment with remaining cardiomyocytes from surviving grafts in our study compared with those reported by others. ${ }^{6-8,12}$ However, the overall effectiveness of cellular grafts is critically linked to various aspects of cell expansion, preservation, and mechanisms of tissue retention and therefore are legitimate targets to explore to improve the efficacy of cellular cardiomyoplasty. Unfortunately, we did not directly study or vary myoblast purities or, for that matter, any other aspects of cell culture and preservation, leading us to cautiously and intentionally avoid directly comparing the efficacy of one cell type or mixture versus another given the large difference in LV dysfunction and physiology in our report compared with that in the work of others. ${ }^{8-12}$

In addition, we cannot rule out the possibility that we did not adequately evaluate systolic function (Table 1), the time of study might have been insufficient, or there were simply an insufficient number of animals studied (the power of these studies was only sufficient to observe a 50\% improvement in either LVEF or $\mathrm{E}_{\mathrm{es}}$ ). We believe, on the basis of our studies, that with more severe LV dilation and dysfunction, longer periods of time or perhaps larger doses of cells might be required for functional changes to be observed.

\section{Remodeling}

The major observation of the present study was the attenuation of LV dilatation after ASM transplantation (Figure 2). Studies in both large and smaller animals have also shown positive effects on LV dilatation after ASM injection. ${ }^{5-8,12}$ However, a novel finding of the current study was that effects on LV dilatation were exclusive for the SA dimension. The mechanism that defines this preferential effect on SA remodeling is not entirely clear. The idea that cellular cardiomyoplasty might be directly affecting scar elasticity and thereby limiting scar expansion is a possible explanation for attenuated regional dilatation. ${ }^{25}$ Although we could not find a measurable improvement in either postsystolic shortening or systolic bulging after ASM injection, the interplay of both in chronically ischemic myocardium has not been well characterized. ${ }^{26}$

If ASM-derived skeletal myofibers can actively resist forces (stretch) in line with their fibers, as demonstrated ex vivo, ${ }^{3}$ and thereby limit LV dilatation, this might also explain the observed attenuation to LV dilatation selectively for the LV SA. For example, as the ventricle becomes increasingly spherical after ischemic injury, the predominate cardiac fiber axis $\left(\mathrm{eg}, 60^{\circ}\right)$ progressively reorients horizontally to the SA $\left(\mathrm{eg}, 30^{\circ}\right) .{ }^{25}$ We provide evidence that ASM-derived skeletal myofibers were found aligned with each other and with the remaining cardiac myocytes, and therefore theoretically the engrafted ASM-derived myofiber orientation would be more aligned with the LV SA. As suggested by our data in a small number of animals, ASMderived myofibers might offer innate resistance to dilatory forces on or along their fiber lengths, thereby selectively preventing dilatation aligned with ASM engraftment along the LV SA (Figure 2).

\section{Study Limitations}

The animal model used in the present study approximates clinical ischemic HF in cause, degree of pathology, and coronary anatomy. ${ }^{13,16,18}$ Microembolization does not fully model the phenomenon of myocardial infarction leading to ischemic HF in all patients, particularly in those patients who have a single large infarct. Moreover, this model greatly accelerates the disease progression typical for chronic ischemic HF. ${ }^{16,27}$

Each animal underwent the same number and types of procedures. Differences found between the groups in the present study could have been a result of the timing of instrumentation (and ASM injection). The fact that attenuated dilatation was observed only in the SA dimension in HF plus ASM animals, whereas LA dilatation was nearly identical between the HF-control and HF plus ASM groups, supports that differences observed between groups could have been dependent on myoblast injection. We have attempted to provide a best estimate of cell survival by using standardized techniques to quantify the number of viable ASM-derived myocytes at 6 weeks so that the relative survival between animals could be compared; however, significant sampling error can exist in the method used to calculate cell survival. ${ }^{28}$ Therefore, values given for cell survival should not be interpreted as absolute but only as a standardized estimate.

Segmental function, regional function, or both, as measured by sonomicrometry, might have not adequately documented function in the exact area of ASM engraftment because of the variability of ASM survival; however, myoblast injection was specifically targeted to and was found in the immediate vicinity of the sonomicrometry crystals at 6 weeks. LV function in the awake animal preparation used in this study, as evaluated through PV analyses, could not be performed at extremely low ventricular volumes because of autonomic activation and inevitable adverse hemodynamic consequences. Therefore, the estimates $\mathrm{E}_{\mathrm{es}}$ and $\mathrm{M}_{\mathrm{w}}$ do not include low-volume measurements. If the methods existed in awake animals to permit an evaluation of function over a wider range of preloads, as possible with an isolated heart preparation, ${ }^{7}$ the possibility exists that a difference could have been found in both the position and slope of these relations.

\section{Conclusions}

The present study describes ASM transplantation in a clinically applicable large-animal model of chronic ischemic 
HF free of concomitant interventions. Despite the apparent lack of direct functional effect on cardiac function in this small group of animals, we were able to demonstrate a significant attenuation in LV dilatation after ASM transplantation. The attenuation in LV dilatation was exclusive to the SA and correlated with an estimate of surviving ASM-derived myocytes. These observations suggest that ASM affects LV remodeling through a mechanism independent of cell-to-cell communication, direct functional improvements, or both, but that ASM engraftment and alignment might play a role in such a mechanism.

We thank Angela Phillips for her expert technical assistance in completing these studies and Kristie Wetzel for histologic assistance.

\section{References}

1. Chiu RCJ, Zibaitis A, Koa RL. Cellular cardiomyoplasty: myocardial regeneration with satellite cell implantation. Ann Thorac Surg. 1995; 60:12-8.

2. Li RK, Jia ZQ, Weisel RD, et al. Cardiomyocyte transplantation improves heart function. Ann Thorac Surg. 1996;62:654-61.

3. Murry CE, Wiseman RW, Schwartz SM, Haushka SD. Skeletal myoblast transplantation for repair of myocardial necrosis. J Clin Invest. 1996;98:2512-23.

4. Scorsin M, Hagege A, Vilquin JT, et al. Comparison of effects of fetal cadiomyocyte and skeletal myoblast transplantation on postinfarction left ventricular function. J Thorac Cardiovasc Surg. 2000;119:116975 .

5. Tambara K, Sakakibara Y, Sakaguchi G, et al. Transplanted skeletal myoblasts can fully replace the infarcted myocardium when they survive in the host in large numbers. Circulation. 2003;108(suppl II):II259-63.

6. Taylor DA, Atkins BZ, Hungspreugs $\mathrm{P}$, et al. Regenerating functional myocardium: improved performance after skeletal myoblast transplantation. Nat Med. 1998;4:929-33.

7. Jain M, DerSimonian H, Brenner DA, et al. Cell therapy attenuated deleterious ventricular remodeling and improves cardiac performance after myocardial infarction. Circulation. 2000;103:1920-7.

8. Ghostine S, Carrion C, Souza LCG, et al. Long-term efficacy of myoblast transplantation on regional structure and function after myocardial infarction. Circulation. 2002;106(suppl I):I131-6.

9. Menasche P, Hagege AA, Scorsin M, et al. Myoblast transplantation for heart failure. Lancet. 2001;357:279-80.

10. Menasche P, Hagege AA, Vilquin JT, et al. Autologous skeletal myoblast transplantation for severe postinfarction left ventricular dysfunction. J Am Coll Cardiol. 2003;41:1078-83.

11. Pagani FD, DerSimonian H, Zawadzka A, et al. Autologous skeletal myoblasts transplanted to ischemia-damaged myocardium in humans. J Am Coll Cardiol. 2003;41:879-88.

12. Pouzet B, Vilquin JT, Hagege AA, et al. Factors affecting functional outcome after autologous skeletal myoblast transplantation. Ann Thorac Surg. 2001;71:844-51.

13. Sabbah HN, Stein PD, Kono T, et al. A canine model of chronic heart failure produced by multiple sequential coronary microembolizations. Am J Physiol. 1991;260:H1379-84.

14. Ryan JB, Hicks M, Cropper JR, et al. The preload recruitable stroke work relationship as a measure of left ventricular contractile dysfunction in porcine allografts. Eur J Cardiothorac Surg. 2002;22:738-45.

15. Kada K, Yasui K, Naruse K, Kamiya K, Kodama I, Toyama J. Orientation change of cardiocytes induced by cyclic stretch stimulation: time dependency and involvement of protein kinases. J Mol Cell Cardiol. 1999;31:247-59.

16. Pfeffer MA, Bruanwald E. Ventricular remodeling after myocardial infarction. Experimental observations and clinical implications. Circulation. 1990;81:1161-72.
17. Atkins BZ, Lewis CW, Kraus WE, Hutcheson KA, Glower DD, Taylor DA. Intracardiac transplantation of skeletal myoblasts yields two populations of striated cells in situ. Ann Thorac Surg. 1999;67:124-9.

18. Menasche P. Myoblast-based cell transplantation. Heart Fail Rev. 2003;8:221-7.

19. Grossman PM, Han Z, Palasis M, Barry JJ, Lederman RJ. Incomplete Retention after Direct Myocardial Injection. Catheter Cardiovasc Interv. 2002;55:392-7.

20. Retuerto MA, Schalch P, Patejunas G, et al. Angiogenic pre-treatment improves the efficacy of cellular cardiomyoplasty performed with fetal cardiomyocyte implantation. J Thorac Cardiovasc Surg. 2004;127:111.

21. Askari A, Unzek S, Goldman CK, et al. Cellular, but not direct, adenoviral delivery of vascular endothelial growth factor results in the improved left ventricular function and neovascularization in dilated ischemic cardiomyopathy. J Am Coll Cardiol. 2004;43:1908-14.

22. Qu Z, Balkir L, van Deutekom JC, Robbins PD, Pruchnic R, Huard J. Development of approaches to improve cell survival in myoblast transfer. J Cell Biol. 1998;142:1257-67.

23. Zhang M, Methot D, Poppa V, Fujio Y, Walsh K, Murry CE. Cardiomyocyte grafting for cardiac repair: graft cell death and anti-death strategies. J Mol Cell Cardiol. 2001;33:907-21.

24. Hutcheson KA, Atkins BZ, Hueman MT, Hopkins MB, Glower DD, Taylor DA. Comparison of benefits on myocardial performance of cellular cardiomyoplasty with skeletal myoblasts and fibroblasts. Cell Transplant. 2000;9:359-68.

25. Torrent-Guasp F, Buckberg GD, Clemente C, et al. The structure and function of the helical heart and its buttress wrapping. Articles I-VII. Semin Thorac Cardiovasc Surg. 2001;13:298-416.

26. Skulstad H, Edvardsen T, Urheim S, et al. Postsystolic shortening in ischemic myocardium, active contraction or passive recoil. Circulation. 2002;106:718-24.

27. Pfeffer MA. Left ventricular remodeling after acute myocardial infarction. Anпи Rev Med. 1995;46:455-66.

28. Abercrombie M. Estimation of nuclear population from microtome sections. Anat Rec. 1946;94:239-47.

\section{Discussion}

Dr Robert C. Robbins (Stanford, Calif). Myocardial restoration has received a lot of recent hype. It has led the US Food and Drug Administration to step in and stop the craziness and stop limiting all these clinical trials. You now are required to get an investigational new drug protocol to do any trials with cell therapy. This is a very important study. First, it was done in large animals. Almost all the data that went from the laboratory to clinical trials were obtained in rodents. That makes this is a very important study. As indicated in the conclusion, it is important in that there was not magical improvement in restoration in function. It just simply prevented dilatation of the heart, sort of a biologic acorn device.

Because cell survival was correlated with function, what factors do you attribute to the low cell survival? Certain things that you did not comment on, such as ischemia, apoptosis, and inflammation, could be factors, but one of the things that I was particularly interested in is 150 to 430 million cells. How was there this much variety in the number of cells? Why in factors related to survival?

Dr McConnell. To the first factor-and that is the Achilles heel of this therapy in general-how do we get more cells to survive this transplantation process? I think in most circumstances about $95 \%$ of the cells are lost within the first 24 hours, and at least with the direct surgical technique, most of those are thought to be lost because of the process of venous and lymphatic clearance during the injection and then leaking back out of the myocardium after the injection because again you are not making a new space. You are simply filling a potential space, and as the remaining myocardium and scar contracts, those cells are exuded and phys- 
ical forces are applied to them. Therefore, there is also probably direct mechanical compromise to those cells. Then there are all of those things that you brought up, such as apoptosis and inflammation, regulating survival that in key instances are likely events. We did not study that directly with these animals but have studies ongoing that are looking at those various aspects. I think cell retention is probably the biggest single hurdle that we need to get over in the therapy.

In this particular study we had one animal that received 1.5 million cells versus the other animals that had up to 400 million cells. There was a portion of one sample that was not available to us. That is why there is a lower number in one instance. As far as the animals that received the higher number, one can-at least in these adult healthy sheep-get these cells to culture up to about a billion within 3 weeks. Now in patients, they are older, with other disease processes, and their myoblasts probably do not have the ability to augment or culture up to that level as quickly.

Dr Robbins. I have one more question. Was there any mitral regurgitation (MR) in these animals? You did not comment on that in your article. And at the end of 6 weeks, even though there was no difference in $\mathrm{LV}$ function and you cannot really do $\mathrm{MVO}_{2}$, but just your observation, could you tell the difference in the animals? Did one group have HF and the other not, or were they all about the same?

Dr McConnell. Qualitatively, I could not tell any difference in the animals. As far as MR, all of these animals had some degree of
MR. We did not systematically study that phenomenon in these animals, but you can tell from our PV data that when you use sonomicrometry, you are obviously instrumenting the ventricle, and therefore you are looking at the volume work that the ventricles are performing. We actually had an enlargement in the stroke volume simply because a portion of that was going toward MR.

Dr Edward Verrier (Seattle, Wash). Dr McConnell, are these the wrong cells? Do we need a more elementary stem cell, or do we need a different environment with different angiogenic factors or growth factors in addition to just the myoblasts for it to work?

Dr McConnell. I would not say just at face value that it did not work. One advantage of these cells is that they already are a fair distance down their lineage toward a myocyte. With more rudimentary cells or pluripotential cells, it is more and more difficult to direct their lineage toward cardiomyogenesis per se. That would be the ideal. Now obviously in the world of stem cells, the paracrine effects of these stem cells, particularly in their ability to effect apoptosis and other processes that might indirectly improve cardiac function, is important; myoblasts might have less effect in that regard. However, there are several camps that are developing this idea, Dr Menashé in Europe has recently discussed this idea of a paracrine effect these myoblasts are having, mainly through hepatocyte growth factor and other mediators. But again, that is to explain the phenomenon that these cells have increased cardiac function that we, in this large animal model, were unable to demonstrate. 


\section{Appendix E1. Skeletal Muscle Biopsy, ASM Culture, and Histology}

Skeletal muscle biopsy specimens (1-3 g) were harvested from the left forelimbs of sheep at the time of the first microembolization in the HF plus ASM group. The forelimb muscle was exposed, and the biopsy specimen was taken by using sharp dissection, avoiding electrocautery, and placed into a tube containing biopsy transport media and shipped to GenVec, Inc (Charlestown, Mass) for ASM preparation and culture similar to that described by Jain and associates (2000).

All cells were expanded for 11 to 12 doublings and cryopreserved before transplantation. The myoblasts were thawed, formulated in transplantation media, and shipped for direct myocardial injection. Myoblast purity was measured by means of reactivity with anti-NCAM mAb (CD56-PE, Clone MY-31; BD Biosciences, San Diego, Calif) and by the ability to fuse into multinucleated myotubes. Cell viability was determined by means of trypan blue exclusion. Myoblasts were loaded into tuberculin syringes (approximately $1.0 \times 10^{8}$ cells $/ \mathrm{mL}$ ) and shipped at $4^{\circ} \mathrm{C}$. At the time of transplantation, cells were allowed to warm slowly to room temperature, resuspended by means of gentle agitation, and injected without further manipulation. ASMs were injected at multiple sites in the infarcted myocardium in proximity to segmental sonomicrometry crystals. To avoid inadvertent intravascular or intraventricular injection of cells, the injection needle was passed into the midmyocardium equidistant from the epicardial and endocardial surfaces over a 3- to 4-cm distance, negative pressure was applied to the syringe, and if no blood was returned, the cells were injected as the needle was slowly withdrawn. Slight pressure was applied over the needle exit site for several seconds after injection to limit cell efflux from the needle track.

After 6 weeks of study, each animal was killed, and the heart was removed and perfused with $10 \%$ buffered formalin. Tissue blocks were made from embolized myocardium receiving ASM injection. Hematoxylin and eosin and Trichrome stains were performed with standard methods.

\section{Immunohistochemistry}

Deparaffinized sections were stained immunohistochemically with an anti-myosin heavy chain antibody that does not react with cardiac muscle, alkaline phosphatase-conjugated MY-32 mAb
(Sigma, St Louis, Mo), to confirm the phenotype of the mature grafts. Sections were developed with BCIP-NBT (Zymed Lab Inc, San Francisco, Calif) and counterstained with nuclear red. Additionally, stains for connexin-43 antibody (Chemicon, Temecula, Calif) and cardiac specific troponin I (Chemicon) were performed.

\section{Estimation of Myoblast Survival}

The heart was cut into blocks approximately $2.5 \mathrm{~cm} \times 2.5 \mathrm{~cm} \times$ $3 \mathrm{~mm}$ in dimension and processed in paraffin. In some cases the whole block was sectioned (5- $\mu \mathrm{m}$ thickness), and in other cases only a portion of the tissue was sectioned. For performing quantitative cell counts, tissue sections were then immunostained for skeletal specific myosin heavy chain (MY-32). Cell viability at 6 weeks was assumed on the basis of the initiation of myosin heavy chain expression (Havenith, 1990), cytoarchitectural organization consistent with skeletal myocytes, and the presence of normalappearing nuclei located peripherally. By using representative tissue sections and computer-assisted imaging analysis, the areas of engraftment were calculated and converted to the number of engrafted nuclei according to a separate count of nuclei density performed on Trichrome-stained sections. The total number of surviving myoblast nuclei in each tissue block was estimated by using the following equation:

Sum of graft area in section $\times$ Density of nuclei per graft area $\times$ No. of sections per block $* \times$ Abercrombie connection

(Abercrombie, 1946).

*Estimated number of sections per block according to approximate block thickness of $3 \mathrm{~mm}$ and section thickness of $5 \mu \mathrm{m}$.

\section{Bibliography}

Abercrombie M. Estimation of nuclear population from microtome sections. Ant Rec. 1946;94:239-47.

Havenith MG, Visser R, Schrijvers-van Schendel JM, Bosman FT. Muscle fiber typing in routinely processed skeletal muscle with monoclonal antibodies. Histochemistry. 1990;93:497-9.

Jain M, DerSimonian H, Brenner DA, et al. Cell therapy attenuated deleterious ventricular remodeling and improves cardiac performance after myocardial infarction. Circulation. 2000; 103:1920-7. 

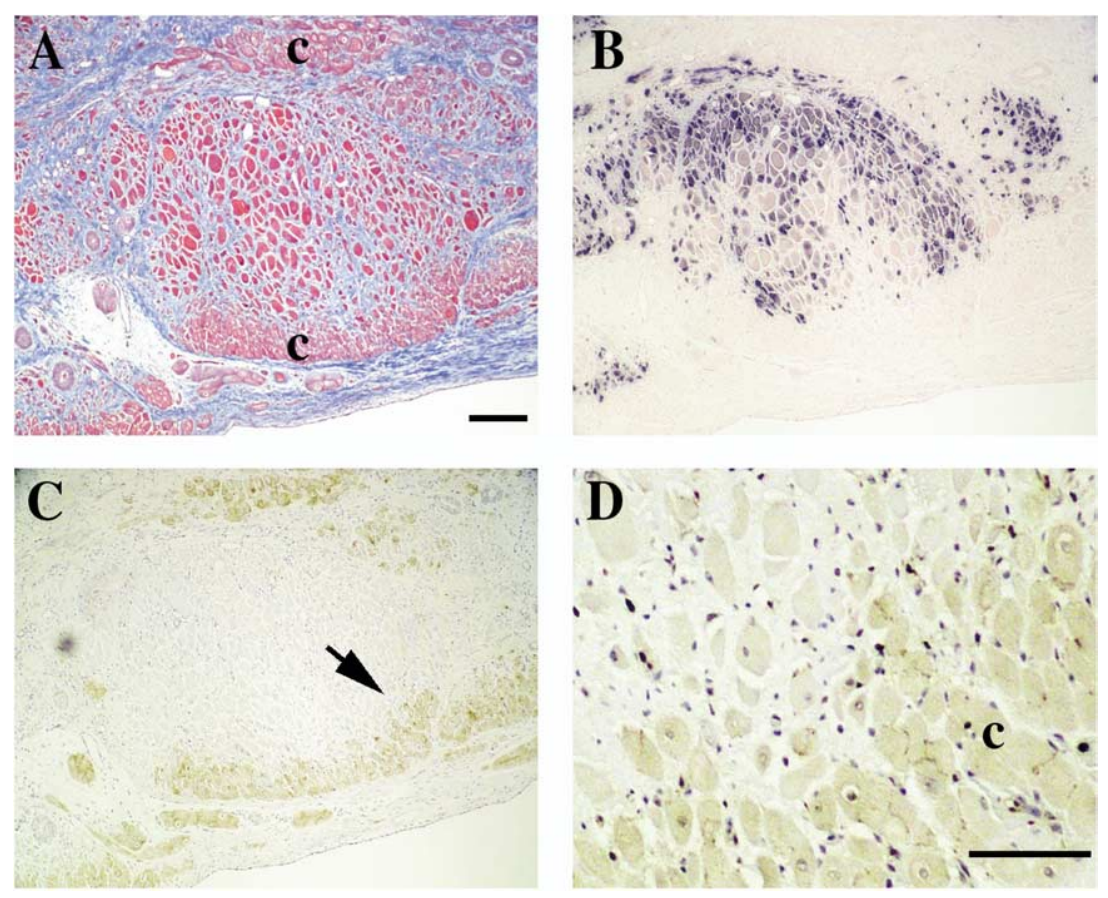

Figure E1. Viable muscle within an area of myocardial fibrosis and scar is seen with Trichrome staining (A). Staining with MY-32 (B) confirmed that ASM-derived skeletal muscle engrafted in close proximity and aligned with remaining cardiac myocytes (c) did not selectively stain for troponin I (C). At higher magnification from the same area (C, arrow), ASM-derived skeletal myocytes do not stain for connexin-43 (D), despite very close apposition to remaining cardiac myocytes (c). Scale bars in panels $A$ and $D$ are $0.2 \mathrm{~mm}$ and $0.1 \mathrm{~mm}$, respectively.

TABLE E1. Left ventricular regional and segmental function after autologous skeletal myoblast transplantation

\begin{tabular}{|c|c|c|c|c|c|}
\hline & $\begin{array}{c}\text { Baseline } \\
(n=6)\end{array}$ & $\begin{array}{c}\text { HF-control, } \\
\text { week } 1 \\
(\mathrm{n}=6)\end{array}$ & $\begin{array}{l}\text { HF-control, } \\
\text { week } 6 \\
(n=6)\end{array}$ & $\begin{array}{c}\text { HF+ASM, } \\
\text { week } 1 \\
(\mathrm{n}=5)\end{array}$ & $\begin{array}{c}\text { HF+ASM, } \\
\text { week } 6 \\
(n=5)\end{array}$ \\
\hline $\mathrm{SA}_{\mathrm{ES}}(\mathrm{mm})$ & $37.9 \pm 2.5$ & $51.8 \pm 2.9^{*}$ & $57.6 \pm 3.6 \dagger$ & $51.5 \pm 4.9$ & $53.4 \pm 4.9$ \\
\hline SA (\% short) & $22.3 \pm 1.2$ & $14.3 \pm 2.3^{*}$ & $13.3 \pm 1.7$ & $15.2 \pm 0.8$ & $13.0 \pm 1.4$ \\
\hline $\mathrm{LA}_{\mathrm{ES}}(\mathrm{mm})$ & $73.2 \pm 3.8$ & $83.7 \pm 5.2^{*}$ & $90.2 \pm 4.6 \dagger$ & $80.4 \pm 4.1$ & $85.5 \pm 4.9$ \\
\hline LA (\% short) & $11.8 \pm 0.9$ & $9.8 \pm 1.1^{*}$ & $8.5 \pm 1.6$ & $8.5 \pm 0.9$ & $8.1 \pm 1.9$ \\
\hline$S L_{\text {post }}(\mathrm{mm})$ & $13.7 \pm 3.4$ & $17 \pm 4.7^{*}$ & $18.9 \pm 5.6$ & $12 \pm 1.3$ & $12.1 \pm 1.3$ \\
\hline$S L_{\text {post }}$ (\% short) & $7.8 \pm 1.6$ & $-1.6 \pm 1.1^{*}$ & $-2.1 \pm 1.8$ & $-1.8 \pm 2.2$ & $-2.4 \pm 1.8$ \\
\hline $\mathrm{SB}(\mathrm{mm})$ & $0.04 \pm 0.03$ & $0.42 \pm 0.10^{*}$ & $0.53 \pm 0.16$ & $0.40 \pm 0.14$ & $0.43 \pm 0.18$ \\
\hline PSS (mm) & $0.06 \pm 0.04$ & $0.47 \pm 0.13^{*}$ & $0.61 \pm 0.2$ & $0.45 \pm 0.13$ & $0.57 \pm 0.12$ \\
\hline
\end{tabular}

Mean \pm standard error of the mean. $H F$, Heart failure; $A S M$, autologous skeletal myoblast; $S A$, left ventricular short axis; $E S$, end systolic; \% short, percentage of systolic shortening; $L A$, left ventricular long axis; $S L_{\text {post }}$ posterior left ventricular segment (microembolized); $S B$, systolic bulging; $P S S$, postsystolic shortening. $* P<.05$ from baseline. $† P<.05$ from week 1 within groups. 

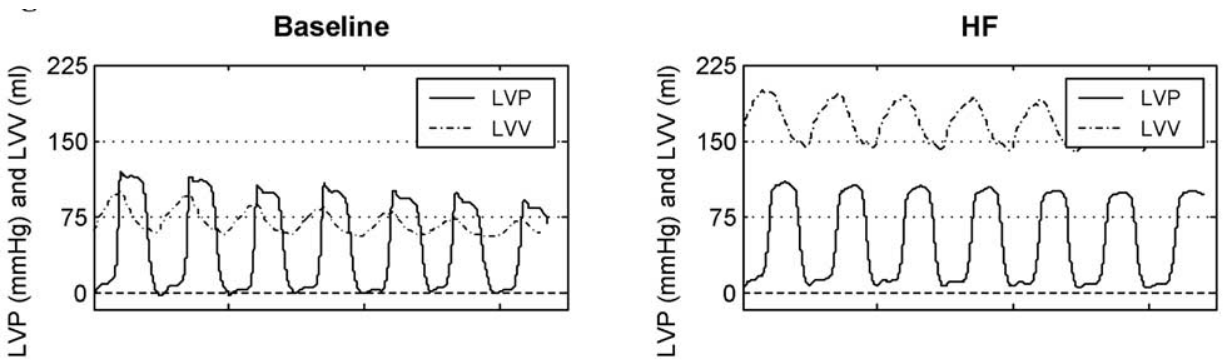

End-Systolic Pressure-Volume Relationships (ESPVR)

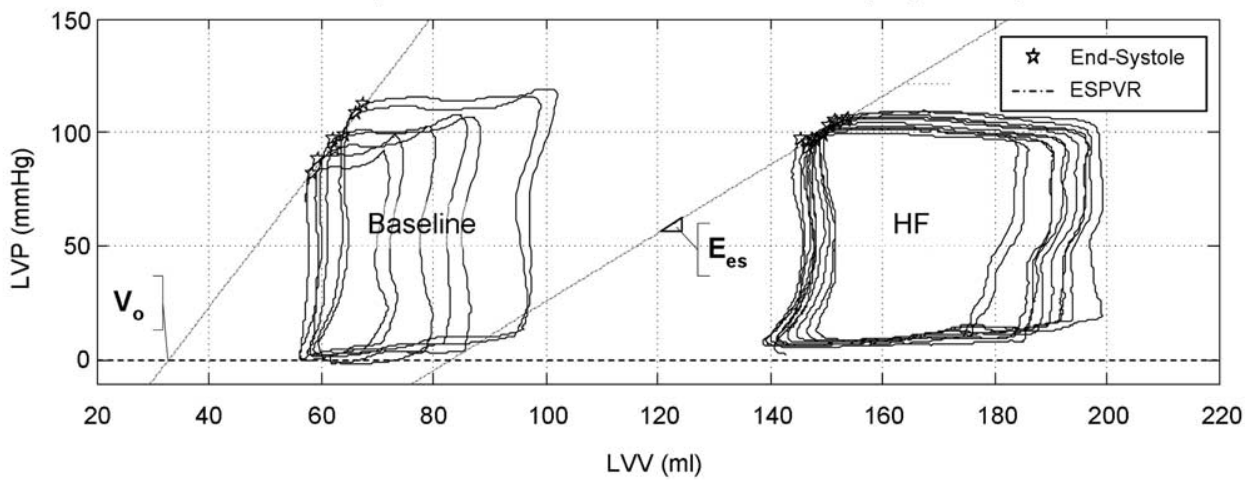

Preload Recruitable Stroke-Work (PRSW)

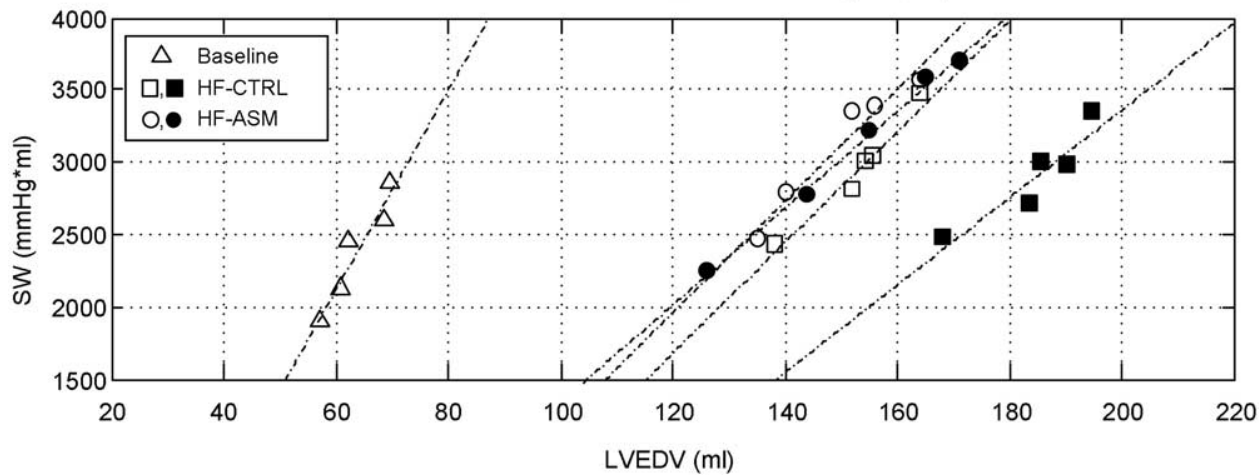

Figure E2. LV volume (LVV) and LV pressure (LVP) tracings from a single sheep before and after microembolization (top and middle panels); highlight changes in the ESPVR (middle) and the PRSW (bottom, squares) with or without ASM transplantation (bottom panel, circles) after microembolization. Although ASM transplantation did not improve cardiac function (slope) after week 1 (open symbols), transplantation did prevent a rightward shift in the PRSW seen in the HF control animal at week 6 (filled symbols). 